\title{
A Mechanism Using Shape-Memory Alloys to Assist Partially Paralysed Patients in Walking With a Natural Gait
}

\author{
Tarana Parvez Kaovasia, Siddharth Dash \\ SRM Institute of Science and Technology \\ Kattankulathur, Chennai, India \\ taranakaovasia_pa@srmuniv.edu.in; siddharthdash_sub@srmuniv.edu.in
}

\begin{abstract}
Shape-Memory Alloys (SMAs) has been gaining impetus in everyday applications, especially in the field of bioengineering. They are used in implants, prosthetics and minimally invasive procedures to name a few. This paper talks about a new mechanism that can be used to help humans who are victims of partial paralysis to walk. The mechanism involved uses a combination of SMAs and piezo-electric sensors to assist these patients to have a natural gait. This mechanism involves wearing a knee-cap, which consists of the SMAs and a boot which consists of the piezoelectric sensors. This is different from the other methods that help a person to walk because the motion while walking is not robotic. Joules heating effect is used to control the shape of the SMA while passive cooling is being used in the circuit to help cool the SMA.
\end{abstract}

Keywords: Shape-Memory Alloy, Piezo-Electric Sensor, Partial Paralysis, Gait.

\section{Introduction}

In the early 1970s, the first large scale application of Shape-Memory Alloys (SMAs) in the industry was as a coupling in Grumaan F-14 aircrafts [1]. These were used as the NiTi couplings shrunk when they were removed from liquid nitrogen. After this, commercialization of SMAs began. In the 1980s, SMAs found orthodontic and other medical applications, mostly because of its super-elasticity but, occasionally due to the Shape Memory Effect (SME) too. It forms the bridge between what the marketplace needs and what the technology provides. In this paper, we aim to use the shape memory effect of the SMA. SMAs are characterized by their innate nature to regain their original shape when a temperature above the transformation (austenite) temperature is reached. This temperature is decided by the chemical composition of the SMA used. The primary aim of this device is to help a patient who has one leg paralyzed (partial paralysis) to walk like any normal person would do. A group of piezo-electric sensors helps detect when the person wants to walk. Further, three different kinds of SMAs are used to help provide ground reaction forces to support the body and provide a periodic movement of the foot; both of which are necessary for walking. The position of the mechanism on the affected leg is shown in Fig. 1. 


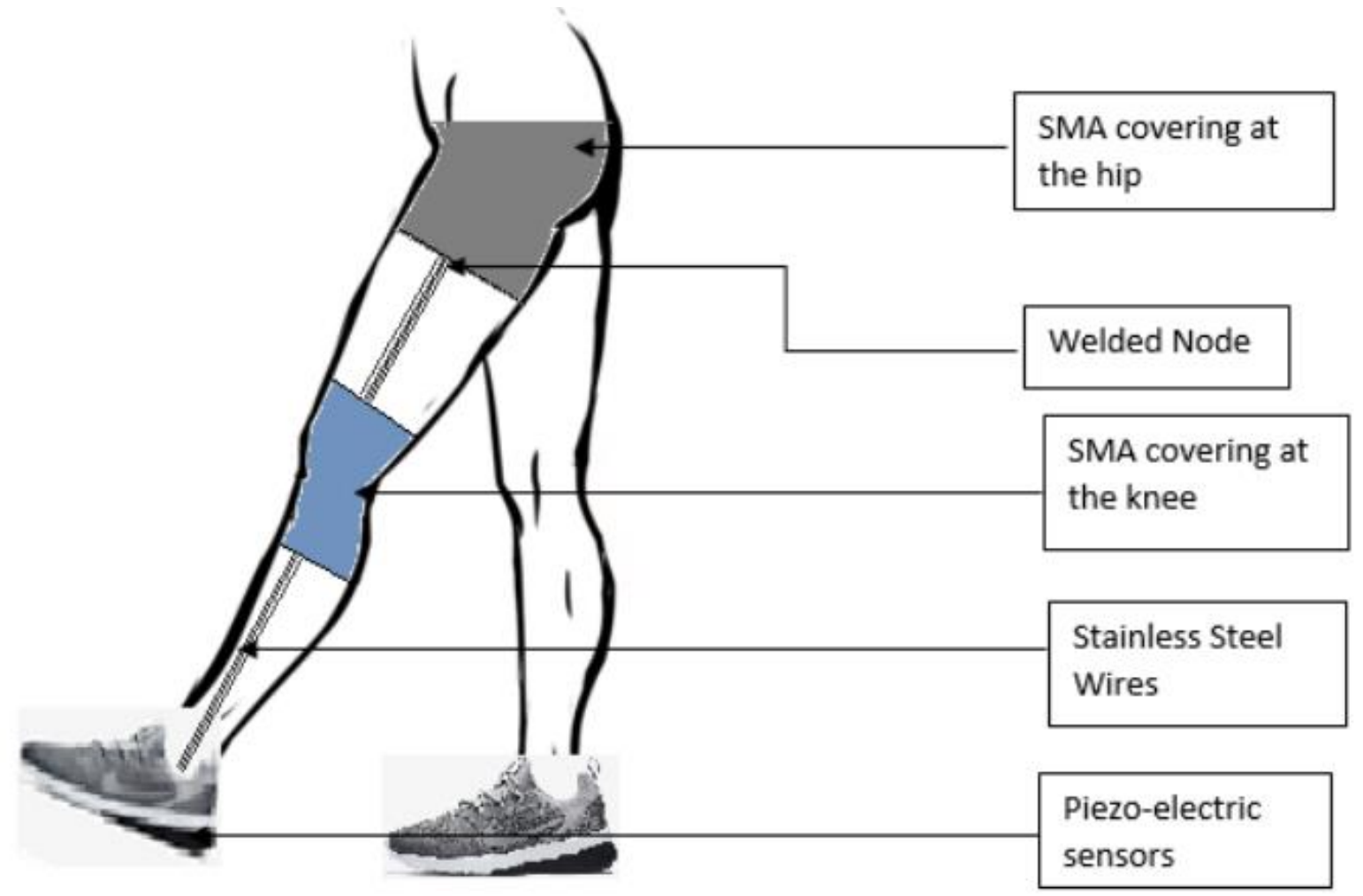

Fig. 1: Affected limb with Mechanism.

\section{Working of the Device}

\subsection{Partwise Working}

\subsubsection{Shape Memory Alloy}

Shape Memory Alloys (SMAs) make use of the Shape Memory Effect to provide a significant use in the functioning as actuators [2]. In the mechanism used, three types of one-way SMAs are employed. These are made of Ni-Ti alloy (Nitinol) but, have different austenite shapes. Using the same material ensures a similar austenite temperature range thus, requiring the same magnitude of actuating current. SMA of type- 1 and type- 2 bring about movement of the knee by about $45^{\circ}$ through alternate actuation. SMA of type- 3 brings about movement of the hip joint by about $25^{\circ}$. SMA of type- 2 and type- 3 are actuated simultaneously to move the leg forward.

These SMAs, shaped like wires, are electrically actuated i.e. heated using Joule's heating effect to reach their austenite temperatures. The wires used have small diameters and are painted black so that cooling can achieved passively. SMA wires of type- 1 and type- 2 are arranged in a semi-circular fashion around the back of the knee. The SMA of type- 3 is located around the outer thigh.

\subsubsection{Piezo-Electric Sensors}

Piezoelectric sensors comprises of a piezoelectric crystal placed between two electrodes. It works on the principle that when a transient pressure is applied on the crystal, there is a change in its mechanical dimensions, resulting in a voltage developing across its surface. When connected in a circuit, current flows through the circuit via the electrodes [3]. The voltage and current generated is proportional to the pressure applied. In the mechanism used, the piezoelectric sensors located in the sole of the shoe are used to aid the microcontroller identify when the individual intends to walk.

\subsubsection{Microcontroller, Logic Gate, Switches}

The microcontroller is the brain of the entire mechanism. It translates the input from the piezoelectric sensors as an intention to walk. When the input from the sensors is above the threshold value, the microcontroller generates a pulse, the width of which is specific to an individual's stride length.

The Switches connect and disconnect the SMAs from the battery on actuation from the microcontroller. 
The Logic Gate, specifically a NOT Gate is used. This ensures SMA of type-2 and type-3 receives a pulse inverted to that received by the SMA of type-1.

\subsubsection{Power Source}

The SMAs will attain its austenite state on reaching its transformation temperature. This state is attained by using a battery as the power source; a source of current which flows through the SMA, heating it and allowing it to attain its transformation temperature by Joules Heating Effect. When current flows through a wire, resistance is offered to this flow of current. This resistance results in generation of heat, which allows the SMAs to reach the required temperature. [4].

\subsubsection{SMA Covering}

\subsubsection{At the Knee}

The knee-cap houses SMA of type-1 and type-2, battery, microcontroller, NOT gate (logic gate) and switches. The inner layer of the knee-cap is made up of an insulating material, which prevents damage to the leg. Meanwhile, the outer layer has good heat transmissibility, thus making it suitable to cool the SMAs passively. The knee-cap has a Velcro belt so that it can be strapped on easily.

\subsubsection{At the Hip}

The SMA of type-3, enclosed in a similar way, is suspended between two Velcro belts, one around the thigh and the other around the waist.

\subsubsection{Wires and Wire Housing}

There are three sets of wires- the first set of wires from the piezoelectric sensors to the microcontroller, the second set of wires from the microcontroller to the SMAs located at the knee and the hip and, finally the SMA wires themselves. The former two sets of wires are composed of stainless steel. A cluster of NiTi SMA wires are joined using tungsten inert gas (TIG) welding process. After this, the NiTi SMA TIG welded node is welded to a stainless-steel wire using laser spot welding process [5]. Any wire outside the SMA covering is separately insulated to provide protection against environmental damage.

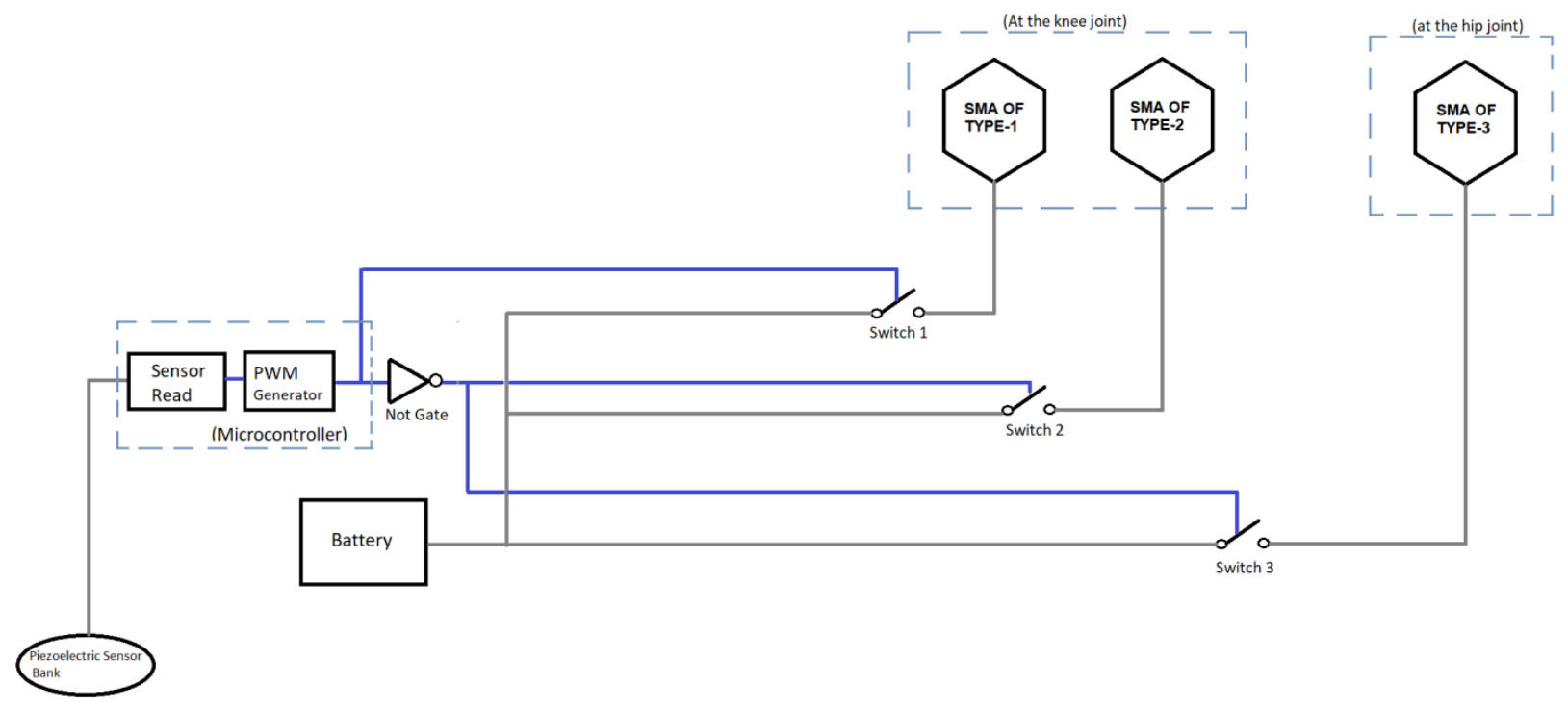

Fig. 2: Working of the Mechanism. 


\subsection{General Working}

Piezoelectric sensors are used as a sensing mechanism to aid the microcontroller in identifying the intention to walk. When the individual is just standing on both feet, weight distribution across the legs is different from when there is an intention to walk. The current generated by the piezoelectric sensors is higher in the latter case. When the microcontroller receives this value of current from the sensor, the actuating mechanism is initiated. The switches connect the SMAs to the power source; a battery, that provides current, which causes the desired heating effect. Because the wires used have less diameter, on removal of current, the rate of dissipation of heat is high and thus there is no requirement of using a heat sink. In addition to this, the wires are painted black to facilitate the heat dissipation. Therefore, when current flows through the SMA wires, the austenite temperature is achieved whereas, on removal of this current, the SMAs undergo passive dissipation of heat. The switches are actuated from the microcontroller using a Pulse Width Modulator (PWM). When the SMAs are actuated via their respective switches, the PWM is directly applied to the SMA of type-1 but there is a NOT Gate between the PWM generator and switches of SMA of type-2 and type-3. Thus, when the PWM is turned on, the pulse reaches its high edge and SMA of type- 1 is actuated and reaches its austenite state, whereas SMA of type- 2 and type- 3 are not actuated. This causes the knee to bend. At this point, the weight is transferred to the healthy foot, which simultaneously moves forward. The next task is to straighten the paralysed leg. This is achieved by actuating SMA of type- 2 and type- 3 when the pulse reaches it low edge. This straightens the paralyzed leg. As long as the microcontroller receives the threshold current from the piezoelectric sensors, the entire actuating process is repeated. If no input is received from the piezoelectric sensors, the SMAs are not actuated. In this way, an individual with a paralysed leg can walk again. The systematic procedure is mapped out with the help of a flow chart depicted in Figure 3.

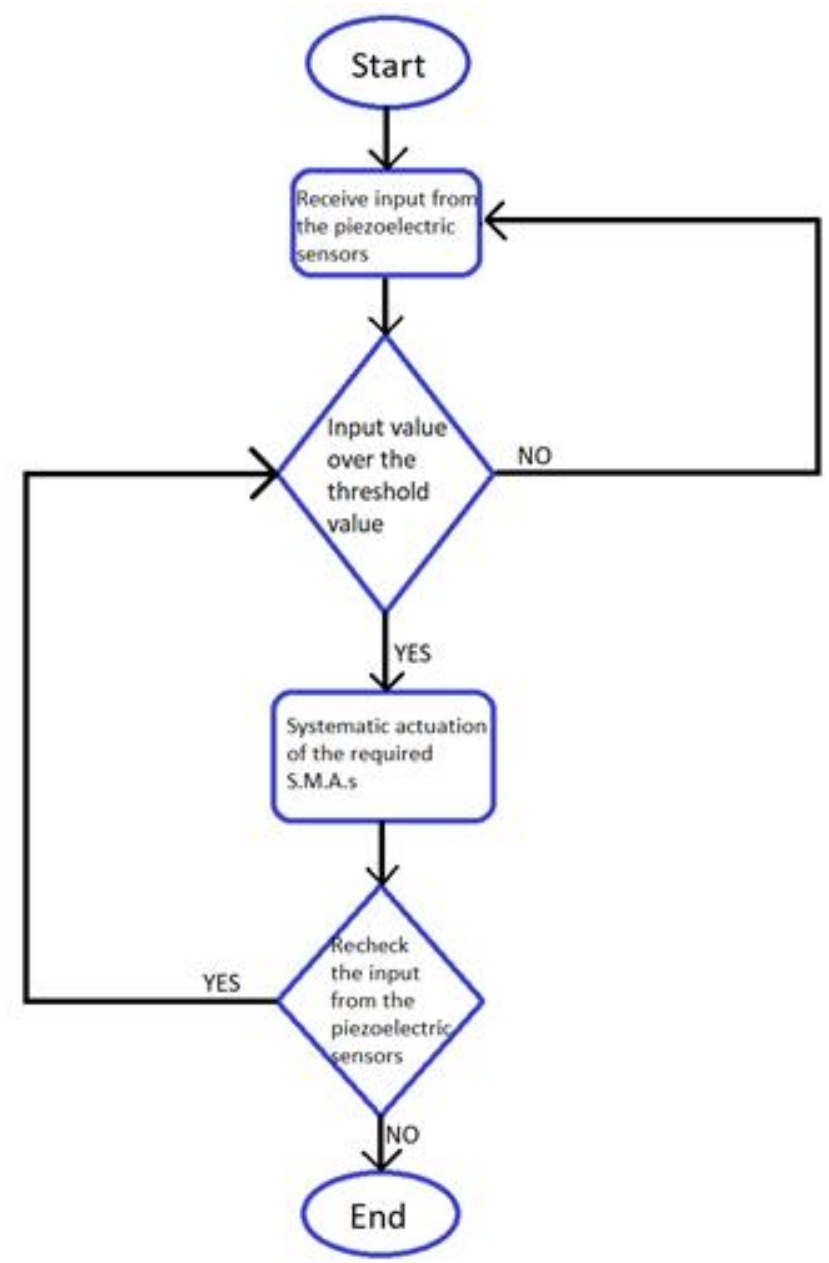

Fig. 3: Flowchart depicting the working of the mechanism. 


\section{Results}

This section discusses about the various results which have been achieved in the paper. They are listed below:

- A theoretical model of the mechanism has been successfully designed to depict the positioning of the mechanism of the affected limb.

- People of different heights exert different pressure on the ground as well as have different stride lengths.

- The mechanism is made custom to a said individual in terms of the threshold value of the piezo-electric transducers set and the width of the pulse as set in the pulse width modulator.

- One-way SMAs have been used which help in movement of the mechanism.

- Passive cooling of the SMAs ensures no additional power consumption.

\section{Conclusion and Discussion}

The mechanism aims to bring about the movement of a paralysed leg in as natural a way as possible. This has been accomplished by using a combination of Piezo-electric sensors, set of SMAs, a microcontroller, NOT gate and switches to work in coordination with each other to make this possible. The proposed mechanism brings about the movement of the leg, which mimics the natural gait of a person. The only source of active power which is provided is a small battery therefore, making the initial expenditure as the only substantial investment into the mechanism. As time progresses, only the batteries need to be replaced. The mechanism uses Velcro belts making it easy for the person to put on and remove the mechanism at will.

\section{Future Work}

This section discusses about the possibility of conducting human trials, so that it can be experimentally verified how well the mechanism mimics the natural gait or not. The tuning of the system is carried out accordingly to perfect the mechanism. Hardware simulations will help calculate the exact dimensions of the various components and help provide a cost budget for the same.

\section{References}

[1] T. Tadaki, K. Otsuka, and K. Shimizu, "Shape memory alloys," Annual Review of Materials Science, vol. 18, no. 1, pp. 25-45, 1988.

[2] H. N. Bhargaw, M. Ahmed, and P. Sinha, "Thermo-electric behaviour of NiTi shape memory alloy," Transactions of Nonferrous Metals Society of China, vol. 23, no. 8, pp. 2329-2335, 2013.

[3] G. Gautschi, Piezoelectric sensorics. Springer Berlin, Heidelberg, New York. 2002, p. 3.

[4] W. Huang, "On the selection of shape memory alloys for actuators," Materials \& design, vol. 23, no. 1, pp. 11-19, 2002.

[5] S.-X. Lue, Z.-L. Yang, and H.-G. Dong, "Welding of shape memory alloy to stainless steel for medical occlude," Transactions of Nonferrous Metals Society of China, vol. 23, no. 1, pp. 156-160, 2013. 